\title{
APPROXIMATE GENERALIZED EXPONENTIAL FUNCTIONS
}

\author{
Eun Hwi LeE
}

ABstract. In this paper we prove the superstability of a generalized exponential functional equation

$$
f(x+y)=a^{2 x y-1} g(x) f(y) .
$$

It is a generalization of the superstability theorem for the exponential functional equation proved by Baker. Also we investigate the stability of this functional equation in the following form :

$$
\frac{1}{1+\delta} \leq \frac{f(x+y)}{a^{2 x y-1} g(x) f(y)} \leq 1+\delta
$$

\section{Introduction}

In 1940, S. M. Ulam gave a wide ranging talk in the Mathematical Club of the University of Wisconsin in which he discussed a number of important unsolved problems (ref. [22]). One of those was the question concerning the stability of homomorphisms :

Let $G_{1}$ be a group and let $G_{2}$ be a metric group with a metric $d(\cdot, \cdot)$. Given $\epsilon>0$, does there exist a $\delta>0$ such that if a mapping $h: G_{1} \rightarrow G_{2}$ satisfies the inequality $d(h(x y), h(x) h(y))<\delta$ for all $x, y \in G_{1}$, then there exists a homomorphism $H: G_{1} \rightarrow G_{2}$ with $d(h(x), H(x))<\epsilon$ for all $x \in G_{1}$ ?

In the next year, Hyers [5] answered the Ulam's question for the case of the additive mapping on the Banach spaces $G_{1}, G_{2}$. Thereafter, the result of

Received July 28, 2009. Revised September 15, 2009.

Key words and phrases : and phrases Exponential functional equation, Stability of functional equation, Superstability.

2000 Mathematics Subject Classification : 39B72, 39B22. 
Hyers has been generalized by Rassias [15]. Since then, the stability problems of various functional equations have been investigated by many authors (see $[1,3,4,6-13,16-21])$.

In particular, Baker et al. in [4] introduced the stability of the exponential functional equation in the following form : if $f$ satisfies the inequality $\mid f(x+$ $y)-f(x) f(y) \mid \leq \varepsilon$, then either $f$ is bounded or $f(x+y)=f(x) f(y)$. This is frequently referred to as Superstability.

In this paper, we will investigate the solution and the superstability of the exponential type functional equation

$$
f(x+y)=a^{2 x y-1} g(x) f(y),
$$

which is a generalization of the superstability of the exponential functional equation given by Baker et al.[2]. Also we investigate the stability of this functional equation in the following form :

$$
\frac{1}{1+\delta} \leq \frac{f(x+y)}{a^{2 x y-1} g(x) f(y)} \leq 1+\delta .
$$

Note that $f(x)=a^{x^{2}+1}$ and $f(x)=a^{x^{2}+x+1}$ are solutions of the equation (1).

\section{Superstability of the generalized exponential functional equa-} tion

Baker et al.[2] proved the superstability of Cauchy's exponential equation

$$
f(x+y)=f(x) f(y)
$$

That is, if the Cauchy difference $f(x+y)-f(x)(y)$ of a real-valued function $f$ defined on a real vector space is bounded for all $x, y$, then $f$ is either bounded or exponential. Their result was generalized by Baker [1] : let $S$ be a semi-group and let $f$ be a complex-valued function defined on $S$ such that

$$
|f(x y)-f(x) f(y)|<\delta
$$


for all $x, y \in S$, then $f$ is either bounded or multiplicative. The following our result is a generalization of Baker's theorem.

Theorem 1. Let $\delta>0$ and $a \geq 1$ be given. Let $f, g:(0, \infty) \rightarrow(0, \infty)$ be functions with $g(m) \geq \max \{2,4 \delta / f(m)\}$ for some integer $m \geq 1$ such that

$$
\left|f(x+y)-a^{2 x y-1} g(x) f(y)\right|<\delta
$$

for all $x, y \in(0, \infty)$. Then

$$
g(x+y)=a^{2 x y-1} g(x) g(y)
$$

for all $x, y \in(0, \infty)$.

Proof. If we replace $x$ and $y$ by $m$ in (2), simultaneously, we get

$$
\left|f(2 m)-a^{2 m^{2}-1} g(m) f(m)\right|<\delta .
$$

By induction, we get that for all $m \geq 2$

$$
\begin{aligned}
& \left|f(n m)-a^{2\left(m^{2}+2 m^{2} \cdots+(n-1) m^{2}\right)-(n-1)} g(m)^{n-1} f(m)\right| \\
& \leq \delta+a^{2(n-1) m^{2}-1} g(m) \delta+a^{2\left((n-1) m^{2}+(n-2) m^{2}\right)-2} g(m)^{2} \delta \\
& +\cdots+a^{2\left((n-1) m^{2}+(n-2) m^{2} \cdots+2 m^{2}\right)-(n-2)} g(m)^{n-2} \delta .
\end{aligned}
$$

In fact, if the inequality (3) holds, we have

$$
\begin{aligned}
& \left|f((n+1) m)-a^{2\left(m^{2}+2 m^{2} \cdots+n m^{2}\right)-n} g(m)^{n} f(m)\right| \\
& \leq\left|f((n+1) m)-a^{2 n m^{2}-1} g(m) f(n m)\right| \\
& +\left|f(n m)-a^{2\left(m^{2}+2 m^{2} \cdots+(n-1) m^{2}\right)-(n-1)} g(m)^{n-1} f(m)\right| a^{2 n m^{2}-1} g(m) \\
& \leq \delta+a^{2 n m^{2}-1} g(m) \delta+a^{2 n m^{2}} a^{(n-1) m^{2}-2} g(m)^{2} \delta \\
& +\cdots+a^{2 n m^{2}} a^{(n-1) m^{2}} \cdots a^{2 m^{2}-n} g(m)^{n-1} \delta
\end{aligned}
$$

for all $n \geq 2$. Note that $2\left(m^{2}+2 m^{2}+\cdots+(n-1) m^{2}\right)-(n-1) \geq 0$ for any $n \geq 1$. By (3), we get

$$
\begin{aligned}
& \left|\frac{f(n m)}{a^{2\left(m^{2}+2 m^{2} \cdots+(n-1) m^{2}\right)-(n-1)} g(m)^{n-1} f(m)}-1\right| \\
& \leq\left(\frac{1}{g(m)^{n-1} f(m)}+\frac{1}{g(m)^{n-2} f(m)}+\cdots+\frac{1}{g(m) f(m)}\right) \delta \\
& <\frac{1}{g(m) f(m)}\left(1+\frac{1}{2}+\frac{1}{2^{2}}+\cdots\right) \delta=\frac{2 \delta}{g(m) f(m)} \leq \frac{1}{2}
\end{aligned}
$$


for all positive integer $n$. Since $g(m)^{n} \rightarrow \infty$ as $n \rightarrow \infty$ and

$$
\begin{gathered}
a^{2\left(m^{2}+2 m^{2} \cdots+(n-1) m^{2}\right)-(n-1)} \geq 1, \\
f(n m) \rightarrow \infty \text { as } n \rightarrow \infty .
\end{gathered}
$$

Then for any $x, y \in(0, \infty)$ we have

$$
\begin{aligned}
& f(n m)\left|g(x+y)-a^{2 x y-1} g(x) g(y)\right| \\
& \leq\left|a^{2 n m(x+y)-1} g(x+y) f(n m)-f(n m+x+y)\right| \frac{1}{a^{2 n m(x+y)-1}} \\
& +\left|f(n m+x+y)-a^{2 x(y+n m)-1} g(x) f(y+n m)\right| \frac{1}{a^{2 n m(x+y)-1}} \\
& +\left|f(y+n m)-a^{2 y n m-1} g(y) f(n m)\right| g(x) \cdot \frac{a^{2 x y}}{a^{2 y n m}}
\end{aligned}
$$

and so

$$
\begin{aligned}
& \left|g(x+y)-a^{2 x y-1} g(x) g(y)\right| \\
& \leq \frac{2 \delta}{f(n m) a^{2 n m(x+y)-1}}+\frac{a^{2 x y} \delta g(x)}{f(n m) a^{2 y n m}} \rightarrow 0
\end{aligned}
$$

as $n \rightarrow \infty$. Thus it follows that

$$
g(x+y)=a^{2 x y-1} g(x) g(y) .
$$

for any $x, y \in 0, \infty)$.

Corollary 2. Let $\delta>0$ and $a \geq 1$ be given. Let $f:(0, \infty) \rightarrow(0, \infty)$ be a function with $f(m) \geq \max \{2,2 \sqrt{\delta}\}$ for some integer $m \geq 1$ such that

$$
\left|f(x+y)-a^{2 x y-1} f(x) f(y)\right|<\delta
$$

for all $x, y \in(0, \infty)$. Then

$$
f(x+y)=a^{2 x y-1} f(x) f(y)
$$

for all $x, y \in(0, \infty)$.

Corollary 3. Let $\delta>0$ and $a \geq 1$ be given. Let $f:(0, \infty) \rightarrow(0, \infty)$ be a function such that

$$
\left|f(x+y)-a^{2 x y-1} f(x) f(y)\right|<\delta
$$


for all $x, y \in(0, \infty)$. Then either $f$ is bounded or

$$
f(x+y)=a^{2 x y-1} f(x) f(y)
$$

for all $x, y \in(0, \infty)$.

Suppose that $H_{p}:(0, \infty) \times(0, \infty) \rightarrow(0, \infty)$ be a monotonically increasing (in both variables) homogeneous mapping, for which $H_{p}(t x, t y)=t^{p} H_{p}(x, y)$ holds for some $p>1$, and for all $t, x, y \in(0, \infty)$. For examples, let $H_{p}(x, y)=$ $a x^{p}+b y^{p}$ for $a, b, x, y \in(0, \infty)$. Then $H_{p}$ is a monotonically increasing (in both variables) homogeneous mapping.

Theorem 4. Let $a \geq 1$ be given. If $f, g:(0, \infty) \rightarrow(0, \infty)$ satisfy the functional inequality

$$
\left|f(x+y)-a^{2 x y-1} g(x) f(y)\right| \leq H_{p}(x, y),
$$

then either $g(x)=o\left(x^{p}\right)$ as $x \rightarrow \infty$ or $g(x+y)=a^{2 x y-1} g(x) g(y)$ for every $x, y \in(0, \infty)$.

Proof. By the same method as the proof of Theorem 1, we have

$$
\begin{aligned}
& \left|f(n x)-a^{2\left(x^{2}+2 x^{2} \cdots+(n-1) x^{2}\right)-(n-1)} g(x)^{n-1} f(x)\right| \\
& \leq H_{p}((n-1) x, x)+H_{p}((n-2) x, x) g(x) a^{2(n-1) x^{2}-1} \\
& +H_{p}((n-3) x, x) g(x)^{2} a^{2\left((n-1) x^{2}+(n-2) x^{2}\right)-2} \\
& +\cdots+H_{p}(x, x) g(x)^{n-2} a^{2\left(2 x^{2}+\cdots+(n-2) x^{2}\right)-(n-2)}
\end{aligned}
$$

for all positive integer $n \geq 2$ and $x>0$. Since $a \geq 1$, we get

$$
\begin{aligned}
& \left|\frac{f(n x)}{g(x)^{n-1} f(x) a^{2\left(x^{2}+2 x^{2}+\cdots+(n-1) x^{2}\right)-(n-1)}}-1\right| \leq \sum_{i=2}^{n-1} \frac{H_{p}(i x, x)}{g(x)^{i-1} f(x)} \\
& \leq \sum_{i=1}^{\infty} \frac{i^{p} H_{p}(x, x)}{g(x)^{i} f(x)} \leq \frac{H_{p}(x, x)}{g(x) f(x)} \sum_{i=1}^{\infty} \frac{i^{p}}{g(x)^{i-1}}
\end{aligned}
$$

for any positive integer $n \geq 2$ and $x>0$. Assume that $g(x) \neq o\left(x^{p}\right)$ as $x \rightarrow \infty$, that is, there exist some $\alpha>0$ and a sequence $\left\{x_{k}\right\}$ in $(0, \infty)$ such 
that $x_{k} \rightarrow \infty$ as $k \rightarrow \infty$ and $g\left(x_{k}\right) \geq \alpha x_{k}^{p}>1$ for sufficiently large $k$. Then $\frac{i^{p}}{g\left(x_{k}\right)^{i-1}} \leq \frac{i^{p}}{\left(\alpha x_{k}^{p}\right)^{i-1}}$ and $\sum_{i=1}^{\infty} \frac{i^{p}}{\left(\alpha x_{k}^{p}\right)^{i-1}}$ converges. We can then let the series $\sum_{i=1}^{\infty} \frac{i^{p}}{g\left(x_{k}\right)^{i-1}}$ converge to a value less than $\frac{g(x) f(x)}{2 H_{p}(x, x)}$ by taking sufficiently large $k$. Thus for some sufficiently large $k$ and any $n \geq 2$, we have

$$
\left|\frac{f\left(n x_{k}\right)}{g\left(x_{k}\right)^{n-1} f(x) a^{-(n-1)} \prod_{i=1}^{n-1} a^{i x_{k}^{2}}}-1\right|<\frac{1}{2} .
$$

By the convergence of $\sum_{i=1}^{\infty} \frac{i^{p}}{g\left(x_{k}\right)^{i-1}}$, we have $\frac{n^{p} H_{p}\left(x_{k}, x_{k}\right)}{g\left(x_{k}\right)^{n-1}} \rightarrow 0$ as $n \rightarrow \infty$. Note that $a^{-(n-1)} \prod_{i=1}^{n-1} a^{i x_{k}{ }^{2}} \geq 1$, we have

$$
\left|\frac{\frac{f\left(n x_{k}\right)}{n^{p} H_{p}\left(x_{k}, x_{k}\right)}}{\frac{g\left(x_{k}\right)^{n-1} f(x) a^{-(n-1)} \prod_{i=1}^{n-1} a^{2 i x_{k}}{ }^{2}}{n^{p} H_{p}\left(x_{k}, x_{k}\right)}}-1\right|<\frac{1}{2}
$$

and so $\frac{n^{p} H_{p}\left(x_{k}, x_{k}\right)}{f\left(n x_{k}\right)} \rightarrow 0$ as $n \rightarrow \infty$.

Now let $x, y>0$ be given. If $k$ is sufficiently large, we have

$$
\begin{aligned}
& f\left(n x_{k}\right)\left|g(x+y)-a^{2 x y-1} g(x) g(y)\right| \\
& \leq\left|a^{2 n x_{k}(x+y)-1} f\left(n x_{k}\right) g(x+y)-f\left(n x_{k}+x+y\right)\right| \frac{1}{a^{2 n x_{k}(x+y)-1}} \\
& +\left|f\left(n x_{k}+x+y\right)-a^{2 x\left(y+n x_{k}\right)-1} g(x) f\left(y+n x_{k}\right)\right| \frac{1}{a^{2 n x_{k}(x+y)-1}} \\
& +\left|f\left(y+n x_{k}\right)-a^{2 y n x_{k}-1} g(y) f\left(n x_{k}\right)\right| \frac{g(x) a^{2 x y-1}}{a^{2 y n x_{k}-1}} \\
& \leq C_{1} H_{p}\left(x+y, n x_{k}\right)+C_{2} H_{p}\left(x, y+n x_{k}\right)+C_{3} H_{p}\left(y, n x_{k}\right) \\
& \leq\left(C_{1}+C_{2}+C_{3}\right) H_{p}\left(n x_{k}, n x_{k}\right)=\left(C_{1}+C_{2}+C_{3}\right) n^{p} H_{p}\left(x_{k}, x_{k}\right)
\end{aligned}
$$

for some $C_{1}, C_{2}, C_{3}>0$ and sufficiently large $n$. Thus

$$
\left|g(x+y)-a^{2 x y-1} g(x) g(y)\right| \leq \frac{\left(C_{1}+C_{2}+C_{3}\right) n^{p} H_{p}\left(x_{k}, x_{k}\right)}{f\left(n x_{k}\right)} \rightarrow 0
$$

as $n \rightarrow \infty$.

Corollary 5. Let $a \geq 1$ be given. If $f:(0, \infty) \rightarrow(0, \infty)$ satisfies the functional inequality

$$
\left|f(x+y)-a^{2 x y-1} f(x) f(y)\right| \leq H_{p}(x, y),
$$

then either $f(x)=o\left(x^{p}\right)$ as $x \rightarrow \infty$ or $f(x+y)=a^{2 x y-1} f(x) f(y)$ for every $x, y \in(0, \infty)$. 


\section{Stability of the equation (1)}

R. Ger [4] introduced a stability for the exponential equation in the following type :

$$
\left|\frac{f(x+y)}{f(x) f(y)}-1\right| \leq \delta .
$$

Now we prove the stability of the equation (1) in a similar type of Ger.

Theorem 6. Let $0<\delta<1$ and $a \geq 1$ be given. If functions $f, g:(0, \infty) \rightarrow$ $(0, \infty)$ satisfies the inequality

$$
\frac{1}{1+\delta} \leq \frac{f(x+y)}{a^{2 x y-1} g(x) f(y)} \leq 1+\delta
$$

for all $x, y \in(0, \infty)$ and $e^{-M} \leq \frac{f(x)}{g(x)} \leq e^{M}$ for some $M$, then there exists $a$ function $F:(0, \infty) \rightarrow(0, \infty)$ such that

$$
F(x+y)=a^{2 x y-1} F(x) F(y)
$$

for all $x, y \in(0, \infty)$,

$$
\frac{1}{1+\delta} \leq \frac{F(x)}{f(x)} \leq(1+\delta) e^{M}
$$

and

$$
\frac{1}{(1+\delta) e^{M}} \leq \frac{F(x)}{g(x)} \leq(1+\delta) e^{2 M}
$$

for all $x \in(0, \infty)$.

Proof. If we define functions $G, H:(0, \infty) \rightarrow(0, \infty)$ by

$$
G(x)=\ln g(x), H(x)=\ln f(x)
$$

for all $x>0$, then the equality (4) may be transformed into

$$
\ln \frac{1}{1+\delta} \leq H(x+y)-\ln a^{2 x y-1}-G(x)-H(y) \leq \ln (1+\delta):=\delta_{1}
$$

and so

$$
\left|H(x+y)-\ln a^{2 x y-1}-G(x)-H(y)\right| \leq \delta_{1}
$$


for all $x, y>0$. By assumption, we have

$$
\left|H(x+y)-\ln a^{2 x y-1}-H(x)-H(y)\right| \leq \delta_{1}+M:=\theta
$$

for all $x, y>0$. Replacing $y$ by $x$ and dividing by 2 , we get

$$
\left|\frac{H(2 x)}{2}-\ln a^{\frac{2 x^{2}-1}{2}}-H(x)\right| \leq \frac{\theta}{2}
$$

for all $x>0$. We use induction on $n$ to prove

$$
\begin{aligned}
& \quad \mid \frac{H\left(2^{n} x\right)}{2^{n}}-\ln a^{\frac{2 x^{2}-1}{2}+\frac{2^{3} x^{2}-1}{2^{2}}+\cdots+\frac{2^{(2 n-1)} x^{2}-1}{2^{n}}-H(x) \mid} \\
& \text { (6) } \leq \theta \sum_{i=1}^{n} \frac{1}{2^{i}}
\end{aligned}
$$

for all $x>0$. On account of (5), the inequality holds for $n=1$. Suppose that inequality (6) holds true for some $n>1$. Then (5) and (6) imply

$$
\begin{aligned}
& \mid \frac{H\left(2^{n+1} x\right)}{2^{n+1}}-\ln a^{\frac{2 x^{2}-1}{2}+\frac{2^{3} x^{2}-1}{2^{2}}+\cdots+\frac{2^{(2 n+1)} x^{2}-1}{2^{n+1}}-H(x) \mid} \\
& \leq \mid \frac{H\left(2^{n} 2 x\right)}{2^{n+1}}-\frac{1}{2} \ln a^{\frac{2(2 x)^{2}-1}{2}+\frac{2^{3}(2 x)^{2}-1}{2^{2}}+\cdots+\frac{2^{(2 n-1)(2 x)^{2}-1}}{2^{n}}-\frac{H(2 x)}{2} \mid} \\
& +\left|\frac{H(2 x)}{2}-\ln a^{\frac{2 x^{2}-1}{2}}-H(x)\right| \\
& \leq \theta \sum_{i=1}^{n+1} \frac{1}{2^{i}},
\end{aligned}
$$

which ends the proof of (6). For any $x>0$ and for every positive integer $n$ we define

$$
P_{n}(x)=\frac{H\left(2^{n} x\right)}{2^{n}}-\ln \prod_{i=1}^{i=n} a^{\frac{2^{(2 i-1)} x^{2}-1}{2^{i}}} .
$$

Let $m, n>0$ be integers with $n>m$. Then it follows from (6)

$$
\begin{aligned}
& \left|P_{n}(x)-P_{m}(x)\right| \\
& =\frac{1}{2^{m}}\left|\frac{H\left(2^{n-m}\left(2^{m} x\right)\right)}{2^{n-m}}-\ln \left(\prod_{i=1}^{n-m} a^{2^{2 i-1}\left(2^{m} x\right)^{2}-1}\right)-H\left(2^{m} x\right)\right| \\
& \leq \theta \sum_{i=m+1}^{n} \frac{1}{2^{i}} \rightarrow 0
\end{aligned}
$$


as $m \rightarrow \infty$. Therefore, the sequence $\left\{P_{n}(x)\right\}$ is a Cauchy sequence, and we may define a function $L:(-\infty, \infty) \rightarrow(-\infty, \infty)$ by

$$
L(x):=\lim _{n \rightarrow \infty} P_{n}(x)
$$

and

$$
F(x):=e^{L(x)}=\lim _{n \rightarrow \infty} \frac{f\left(2^{n} x\right)^{\frac{1}{2^{n}}}}{\prod_{i=1}^{i=n} a^{\frac{2^{(2 i-1)} x^{2}-1}{2^{i}}}}
$$

for all $x>0$. Thus

$$
\begin{aligned}
& \frac{F(x+y)}{a^{2 x y-1} F(x) F(y)} \\
& =\lim _{n \rightarrow \infty} \frac{f\left(2^{n} x+2^{n} y\right)^{\frac{1}{2^{n}}} \prod_{i=1}^{i=n} a^{\frac{2^{(2 i-1)} x^{2}-1}{2^{i}}} \prod_{i=1}^{i=n} a^{\frac{2^{(2 i-1)} y^{2}-1}{2^{i}}}}{a^{2 x y-1} f\left(2^{n} x\right)^{\frac{1}{2^{n}}} f\left(2^{n} y\right)^{\frac{1}{2^{n}}} \prod_{i=1}^{i=n} a^{\frac{2^{(2 i-1)}(x+y)^{2}-1}{2^{i}}}} \\
& =\lim _{n \rightarrow \infty}\left[\frac{f\left(2^{n} x+2^{n} y\right)}{f\left(2^{n} x\right) f\left(2^{n} y\right)}\right]^{\frac{1}{2^{n}}} \cdot \frac{a^{-\left(\frac{1}{2}+\frac{1}{2^{2}}+\cdots+\frac{1}{2^{n}}\right)}}{a^{2 x y-1} a^{2 x y\left(1+2+\cdots+2^{n-1}\right)}} \\
& =\lim _{n \rightarrow \infty}\left[\frac{f\left(2^{n} x+2^{n} y\right)}{a^{2\left(2^{n} x\right)\left(2^{n} y\right)-1} f\left(2^{n} x\right) f\left(2^{n} y\right)}\right]^{\frac{1}{2^{n}}}
\end{aligned}
$$

and

$$
\begin{aligned}
& \left(\frac{1}{1+\delta}\right)^{\frac{1}{2^{n}}} \leq\left[\frac{f\left(2^{n} x+2^{n} y\right)}{a^{2\left(2^{n} x\right)\left(2^{n} y\right)-1} g\left(2^{n} x\right) f\left(2^{n} y\right)}\right]^{\frac{1}{2^{n}}} \\
& \leq\left[\frac{f\left(2^{n} x+2^{n} y\right)}{a^{2\left(2^{n} x\right)\left(2^{n} y\right)-1} f\left(2^{n} x\right) f\left(2^{n} y\right)}\right]^{\frac{1}{2^{n}}}\left(e^{M}\right)^{\frac{1}{2^{n}}} \\
& \leq\left[\frac{f\left(2^{n} x+2^{n} y\right)}{a^{2\left(2^{n} x\right)\left(2^{n} y\right)-1} g\left(2^{n} x\right) f\left(2^{n} y\right)}\right]^{\frac{1}{2^{n}}}\left(e^{2 M}\right)^{\frac{1}{2^{n}}} \\
& \leq(1+\delta)^{\frac{1}{2^{n}}}\left(e^{2 M}\right)^{\frac{1}{2^{n}}}
\end{aligned}
$$

for all $x, y>0$ and for every positive integer $n$. Therefore we have

$$
F(x+y)=a^{2 x y-1} F(x) F(y)
$$

for all $x, y>0$. We can easily see from (4) that

$$
\begin{aligned}
& \left(\frac{1}{1+\delta}\right)^{\frac{1}{2}} \leq \frac{f(2 x)^{\frac{1}{2}}}{a^{\frac{2 x^{2}-1}{2}} g(x)^{\frac{1}{2}} f(x)^{\frac{1}{2}}} \leq \frac{f(2 x)^{\frac{1}{2}}}{a^{\frac{2 x^{2}-1}{2}} f(x)} \cdot e^{\frac{M}{2}} \\
& \leq \frac{f(2 x)^{\frac{1}{2}}}{a^{\frac{2 x^{2}-1}{2}} f(x)^{\frac{1}{2}} g(x)^{\frac{1}{2}}} \cdot e^{\frac{2 M}{2}} \leq(1+\delta)^{\frac{1}{2}} \cdot e^{\frac{2 M}{2}}
\end{aligned}
$$


for all $x>0$. Note that for all $x>0$ and for every positive integer $n$

$$
\begin{aligned}
& \frac{f\left(2^{n} x\right)^{\frac{1}{2^{n}}}}{\prod_{i=1}^{i=n} a^{\frac{2^{(2 i-1)} x^{2}-1}{2^{i}}} f(x)} \\
& =\frac{f\left(2^{n} x\right)^{\frac{1}{2^{n}}}}{\left(a^{\left(2\left(2^{n-1} x\right)^{2}-1\right.}\right)^{\frac{1}{2^{n}}} f\left(2^{n-1} x\right)^{\frac{1}{2^{n-1}}}} \cdot \frac{f\left(2^{n-1} x\right)^{\frac{1}{2^{n-1}}}}{\left(a^{2\left(2^{n-2} x\right)^{2}-1}\right)^{\frac{1}{2^{n-1}}} f\left(2^{n-2} x\right)^{\frac{1}{2^{n-2}}}} \\
& \cdots \frac{f(2 x)^{\frac{1}{2}}}{\left(a^{2 x^{2}-1}\right)^{\frac{1}{2}} f(x)} .
\end{aligned}
$$

Thus we have

$$
\begin{aligned}
\left(\frac{1}{1+\delta}\right)^{\frac{1}{2}+\frac{1}{2^{2}}+\cdots+\frac{1}{2^{n}}} & \leq \frac{f\left(2^{n} x\right)^{\frac{1}{2^{n}}}}{a^{\frac{x^{2}}{2}} a^{x^{2}} \cdots a^{2^{n-2} x^{2}} f(x)} \\
& \leq\left((1+\delta) e^{M}\right)^{\frac{1}{2}+\frac{1}{2^{2}}+\cdots+\frac{1}{2^{n}}}
\end{aligned}
$$

and so

$$
\frac{1}{1+\delta} \leq \frac{F(x)}{f(x)} \leq(1+\delta) e^{M},
$$

and thus

$$
\frac{1}{(1+\delta) e^{M}} \leq \frac{F(x)}{g(x)} \leq(1+\delta) e^{2 M}
$$

for all $x>0$.

Corollary 7. Let $0<\delta<1$ and $a \geq 1$ be given. If a function $f:(0, \infty) \rightarrow$ $(0, \infty)$ satisfies the inequality

$$
\frac{1}{1+\delta} \leq \frac{f(x+y)}{a^{2 x y-1} f(x) f(y)} \leq 1+\delta
$$

for all $x, y \in(0, \infty)$, then there exists a function $F:(0, \infty) \rightarrow(0, \infty)$ such that

$$
F(x+y)=a^{2 x y-1} F(x) F(y)
$$

for all $x, y \in(0, \infty)$ and

$$
\frac{1}{1+\delta} \leq \frac{F(x)}{f(x)} \leq(1+\delta)
$$

for all $x \in(0, \infty)$. 


\section{References}

1. J. Baker, The stability of the cosine equations, Proc. Amer. Math. Soc. 80 (1980), 411-416.

2. J. Baker, J. Lawrence and F. Zorzitto, The stability of the equation $f(x+y)=f(x)+f(y)$, Proc. Amer. Math. Soc. 74 (1979), 242-246.

3. G. L. Forti, Hyers-Ulam stability of functional equations in several variables, Aequationes Math. 50 (1995), 146-190.

4. R. Ger, Superstability is not natural, Rocznik Naukowo-Dydaktyczny WSP Krakkowie, Prace Mat. 159 (1993), 109-123.

5. D.H. Hyers, On the stability of the linear functional equation, Proc. Nat. Acad. Sci. U. S. A. 27 (1941), 222-224.

6. D.H. Hyers and Th.M. Rassias, Approximate homomorphisms, Aequatioues Math. 44 (1992), 125-153.

7. D.H. Hyers, G. Isac, and Th.M. Rassias, Stability of functional equations in several variables, Birkhäuser-Basel-Berlin(1998).

8. K.W. Jun, G.H. Kim and Y.W. Lee, Stability of generalized gamma and beta functional equations, Aequation Math. 60(2000), 15-24.

9. S.-M. Jung, On the general Hyers-Ulam stability of gamma functional equation, Bull. Korean Math. Soc. 34 No 3 (1997), 437-446.

10. S.-M. Jung, On the stability of the gamma functional equation, Results Math. 33 (1998), 306-309.

11. G.H. Kim, and Y.W. Lee, The stability of the beta functional equation, Babes-Bolyai Mathematica, XLV (1) (2000), 89-96.

12. Y.W. Lee, On the stability of a quadratic Jensen type functional equation, J. Math. Anal. Appl. 270 (2002) 590-601.

13. Y.W. Lee, The stability of derivations on Banach algebras, Bull. Institute of Math. Academia Sinica, 28 (2000), 113-116.

14. Y.W. Lee and B.M. Choi, The stability of Cauchy's gamma-beta functional equation, J. Math. Anal. Appl. 299 (2004), 305-313.

15. Th.M. Rassias, On the stability of the linear mapping in Banach spaces, Proc. Amer. Math. Soc. 72 (1978), 297-300.

16. Th.M. Rassias, On a problem of S. M. Ulam and the asymptotic stability of the Cauchy functional equation with applications, General Inequalities 7. MFO. Oberwolfach. Birkhäuser Verlag. Basel ISNM Vol 123 (1997), 297-309.

17. Th.M. Rassias, On the stability of the quadratic functional equation and its applications, Studia. Univ. Babes-Bolyai XLIII(3). (1998), 89-124.

18. Th.M. Rassias, The problem of S. M. Ulam for approximately multiplication mappings, J. Math. Anal. Appl. 246 (2000), 352-378. 
19. Th.M. Rassias, On the stability of functional equation in Banach spaces, J. Math. Anal. Appl. 251 (2000), 264-284.

20. Th.M. Rassias, On the stability of functional equations and a problem of Ulam, Acta Applications. Math. 62 (2000), 23-130.

21. Th.M. Rassias and P. Semrl, On the behavior of mapping that do not satisfy Hyers-Ulam stability, Proc. Amer. Math. soc. 114 (1992), 989-993.

22. S.M. Ulam, Problems in Modern Mathematics, Proc. Chap. VI. Wiley. NewYork, 1964.

\section{Eun Hwi Lee}

Department of Matematics

Jeonju University

Jeonju 560-759, Korea

E-mail: ehl @ jj.ac.kr 